\title{
Los servicios de valor añadido en los portales periodísticos: una propuesta de clasificación
}

\author{
Rosana López Carreño \\ José Vicente Rodríguez Muñoz \\ Departamento de Información y Documentación \\ Universidad de Murcia
}

\section{1. Resumen}

Propuesta de clasificación de los servicios de valor añadido (SVA) de los portales periodísticos españoles a partir del análisis de un total de diecisiete portales, los de los rotativos nacionales y una representación de la prensa regional. Se identificaron ocho categorías de SVA: acceso a la información, interactivos, participación, multimedia, personalizados, entretenimiento, comerciales y complementarios. Los SVA más consolidados son los pertenecientes a las categorías de acceso a la información e interactivos, seguidos muy de cerca por los de participación. Los portales periodísticos que destacan en la categoría de SVA de acceso a la información son los pertenecientes a los diarios El Mundo, El País y La Verdad. En el caso de los SVA interactivos sobresale ABC y Marca, y con respecto a los SVA de participación destaca La Vanguardia.

Palabras clave: Periodismo electrónico. Portal periodístico. Producto documental. Producto informativo. Servicios de valor añadido.

\section{2. Abstract}

Proposal of classification of the added value services (AVS) in the portals of the Spanish journals. Seventeen portals were analysed: all of the national journals and a representation of the regional press. Eight categories were detected: information access, interactive, sharing, multimedia, personalized, amusement, commercial and complementary. The more consolidated AVS were information access and interactive, followed short by information sharing ones. The journals leading in the information access AVS were El Mundo, El País y La Verdad; in interactive AVS, ABC and Marca; and in information sharing, La Vanguardia.

Keywords: Added value services. Digital journalism. Reference products. Informative products. Media portals. 


\section{Introducción}

Desde la aparición, en la década de los años setenta, de los primeros servicios informativos basados en la transmisión de textos, el proceso evolutivo de los sistemas de comunicación ha sido vertiginoso, al mismo tiempo que imprescindible para la supervivencia de la tarea periodística en la incipiente Sociedad de la Información a la que se encuentra abocada.

En sus inicios, el empleo de lo electrónico en el periodismo estaba más orientado a la edición y almacenamiento que a la difusión. Con la aparición de Internet, los medios de comunicación reconocieron de manera inmediata su potencialidad. Así, a principios de los años noventa, se llevan a cabo en Estados Unidos las primeras incursiones del periodismo en Internet, propulsadas por el medio de comunicación social presuntamente más amenazado por la era digital, la prensa y que, curiosamente ha alcanzado en el momento actual un nivel de desarrollo mayor que la radio o la televisión en este nuevo entorno. Con Internet, el denominado periodismo electrónico se caracteriza fundamentalmente por su "multimedialidad, interactividad, renovación constante y acceso desde cualquier lugar del mundo" (Díaz, 1997).

Inicialmente, en este nuevo escenario comunicacional, surgieron las versiones web de los distintos tipos de medios de comunicación social ya consolidados. Una parte importante de estas web han evolucionado hasta convertirse en sitios de la red que no sólo ofrecen información periodística de actualidad, sino que también proporcionan un valor extra a ese conjunto de informaciones, con atractivos reclamos para sus potenciales lectores, oyentes o televidentes, y alcanzando la categoría de verdaderos portales. Estos sitios informativos, los llamados portales periodísticos, conjugan dinámicamente los tres elementos esenciales para su consolidación -el contenido, los productos y los servicios - ; y se constituyen como nuevos medios de comunicación, autónomos y multimediáticos, gracias al vehículo universal que los sustenta, Internet.

\section{Los medios de comunicación en Internet}

Internet es un medio de comunicación en el sentido de cauce o vía de acceso pero no constituye un medio de comunicación social en sí mismo, al carecer de carácter periodístico. Los servidores remotos conectados a la red son los nuevos soportes de la información periodística, como durante tanto tiempo lo ha sido el papel para el periodismo impreso (Pablos, 2000). La aparición de estos nuevos soportes, que simultáneamente conjugan texto, audio y vídeo en la red, permitió el surgimiento de las versiones web de los distintos tipos de medios de comunicación social, tal como se ha indicado anteriormente, encabezados por la prensa, que fue el primer medio de comunicación convencional en ofrecer versión web. El grado de implantación de estos medios en la web es variado. Algunos son verda-

Scire. $9: 2$ (jul.-dic. 2003) 111-122. 
deros portales, mientras que otros aún no acaban de encontrar su función dentro de Internet, y se limitana proporcionar información referencial sobre el medio convencional del cual derivan, siguiendo un proceso de desarrollo más lento. Esto se justifica, fundamentalmente, por las dificultades tecnológicas y técnicas — velocidad de las redes, capacidad de los servidores, sistemas de gestión, etc. - , las dificultades económicas - normalmente en los casos de medios modestos no pertenecientes a los grandes grupos de comunicación-, las dificultades políticas - escasez de licencias, políticas de empresa, etc. - y la falta de visión de la dirección del medio.

En la tabla I se clasifican los sitios en Internet de los medios de comunicación según su origen y tipología.

\begin{tabular}{|c|c|}
\hline ORIGEN & TIPOLOGÍA \\
\hline Medios Textuales & Diarios. Revistas. \\
\hline Radio & Emisoras. Cadenas. Programas. \\
\hline Televisión & Emisoras. Estudios. Programas. \\
\hline Otros & $\begin{array}{l}\text { Agencias de comunicación. } \\
\text { Grupos de comunicación. }\end{array}$ \\
\hline
\end{tabular}

Tabla I. Tipología de sitios de medios de comunicación en Internet

En el caso de los medios de origen textual, son los diarios frente a las revistas los que se preconizan, mayoritariamente, como portales periodísticos, con independencia del ámbito que abarquen (nacional, regional o local) y del tipo de información que traten (general o especializada). Ejemplos de portales de diarios son $A B C$, El País, El Mundo, La Vanguardia, Marca, Expansión y un numeroso etcétera.

En cuanto a las revistas, cabe decir que aún no han llegado a conformarse como portales, aunque sí representan una valiosa fuente informativa. Algunos ejemplos son las revistas de información general como Época o Tiempo de hoy; las revistas del corazón como Hola, Diez Minutos y Lecturas; además de revistas femeninas, de Informática, científicas y otras de carácter especializado.

Respecto a los sitios web de emisoras y cadenas de radio en línea, hay que señalar que han evolucionado de manera vertiginosa en el último año, pasando de ser simples páginas web informativas sobre el medio convencional del que procedían a convertirse en portales periodísticos con una variada oferta de productos y servicios para sus ciber-oyentes, que permiten tanto la conexión en directo de la emisión convencional (ondas) como la audición de programas en diferido. 
Ejemplos, entre otros, de radios en línea son Cadena 100, COPE, Los 40 principales, Onda Cero, Radio Nacional de España, Ser, etcétera.

Las televisiones en línea también se encuentran en pleno desarrollo de sus respectivos portales, pero en menor medida que la radio, dado que éstas no ofrecen aún la conexión en línea a sus emisiones convencionales, sino que el usuario se conecta al servidor y descarga un vídeo, normalmente correspondiente a avances informativos y de duración reducida. En ambos casos, esta progresión no alcanza los niveles obtenidos por los portales periodísticos de origen textual (prensa). Como ejemplos de la televisión en línea destacan RTVE, Antena 3, Tele 5 , o Canal + .

En cuanto a los sitios en la red sostenidos por agencias de comunicación o de noticias, éstos están más orientados a los profesionales de la comunicación que al público en general; ya que, a pesar de ofrecer información de actualidad de forma gratuita, algunos de los servicios específicos que ofrecen - como la obtención de datos y de informaciones elaboradas - tienen acceso restringido. Ejemplos de estos sitios en lo que respecta a agencias generalistas tenemos las agencias Efe y Europa Press, y en cuanto a agencias especializadas, como las fotográficas encontramos la agencia Cover. Los sitios web sostenidos por los grupos de comunicación suelen ser páginas de información referencial sobre los miembros del grupo, proporcionando información general sobre ellos y estableciendo acceso a los respectivos sitios de los mismos. Muestra de ello son el Grupo Correo, Grupo Godó, Grupo Prisa o el Grupo Zeta, entre otros.

\section{Taxonomía de los portales periodísticos}

La definición de portal viene dada por la concentración de información y servicios en un mismo sitio web. Es, por lo tanto, un concepto mucho más amplio que el de una página o un conjunto de páginas web alojadas en un servidor. Muchos autores suelen mencionar el término portal informativo para referirse a los portales de los medios de comunicación. En este caso, el uso de la palabra informativo parece redundante, ya que si se entiende que la información es uno de los pilares sobre los que descansa la naturaleza de un portal, resultaría más apropiado hablar de portales de medios de comunicación para referirnos a los portales vinculados a los medios de comunicación social tradicionales o a los desarrollados específicamente para la web.

El impacto de Internet en las relaciones entre las organizaciones y sus clientes, desemboca en un aumento de aquéllas; de manera que ya no se trata exclusivamente de vender un producto, sino de proporcionar también toda una serie de servicios. Braa, Sorensen y Dahlbom proponen como ejemplo típico de este proceso evolutivo al portal periodístico, que ellos entienden como un género

Scire. $9: 2$ (jul.-dic. 2003) 111-122. 


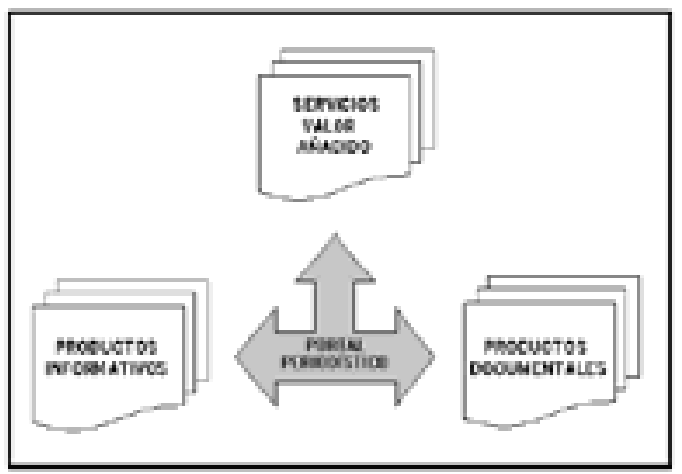

Figura1. Elementos básicos de los portales periodísticos

particular de los portales de Internet y, al mismo tiempo, como una modalidad de periódico. En este caso el producto original - el diario - viene acompañado de una serie de servicios de valor añadido (Braa et al., 2000). La figura 1 muestra los elementos básicos que conforman la tipología de portal periodístico, siendo el eje principal el contenido, que es canalizado a través de los productos informativos, productos documentales y servicios de valor añadido.

Desde la perspectiva del portal periodístico, se considera al producto informativo como aquel producto vinculado directamente a la unidad básica - la noticia - y al producto documental como continente de información tratada y organizada, no necesariamente vinculada a la noticia, cuya finalidad es satisfacer una necesidad informativa definida a priori. Es decir, el producto informativo se constituye como un refuerzo de la noticia, mientras que el producto documental refuerza otro tipo de informaciones. Sin embargo, en muchas ocasiones estos conceptos se entremezclan, resultando difícil su delimitación, por existir una frontera difusa entre ellos (López, 2002).

En la tabla II, se recoge una clasificación de los productos informativos y productos documentales más frecuentes en los portales periodísticos. Es evidente que los productos informativos se encuentran en un grado de desarrollo más avanzado frente a los productos documentales, dado que estos últimos tienen una vinculación más afín al profesional documentalista (gestor de contenidos), figura que aún no ha alcanzado la valoración que le corresponde en la empresa periodística. Pero el elemento más consolidado y frecuente en los portales periodísticos es el servicio de valor añadido, debido principalmente a su amplia gama y popularidad entre los usuarios, además de ser el elemento más diferenciador entre ellos. 


\begin{tabular}{|c|c|}
\hline Productos informativos & Productos documentales \\
\hline Noticias & FAQs \\
\hline Especiales & Agenda \\
\hline Suplementos & Guías de recursos \\
\hline Edición impresa & Guías turísticas \\
\hline Entrevistas & Callejeros \\
\hline Programación TV y radio & Asesoría \\
\hline Tiempo & Empleo \\
\hline Loterías & Resúmenes de noticias \\
\hline
\end{tabular}

Tabla II. Productos informativos y documentales de los portales periodísticos

\section{Los servicios de valor añadido (SVA)}

Los SVA son servicios que se elaboran o prestan con un alto grado de correspondencia respecto a las necesidades informativas de los usuarios (Valdés, 1999). Es por ello que el suministro de servicios con valor añadido requiere una mayor elaboración. Para Fuentes i Pujol son los elementos ofertados en los portales "que a las versiones en papel les sea técnica y físicamente imposible ofrecer" (ref. Moreiro, 2000). La mayoría de los portales periodísticos ofrecen una amplia gama de contenidos gratuitos con el objetivo de atraer y mantener el tráfico hacia su web y complementar así la originaria oferta de la edición impresa. El valor añadido representa, por tanto, un registro opcional, de pago o gratuito, a partir del cual el usuario requiere una atención personal por esa aportación económica o información suministrada (Palazón, 2001).

En este contexto, los SVA se identifican como aquellos servicios que resaltan sobre la oferta de servicios comunes de los portales, en general, por implicar un valor extra gracias a las herramientas que Internet posibilita. Parece claro que lo que hoy se considera SVA probablemente mañana será un servicio común. Es decir, su consideración como tal SVA depende de la condición espacio-tiempo en la que emergen en Internet, y que los portales periodísticos - entre ellos los diarios electrónicos - adaptan para aumentar sus prestaciones.

Fuentes i Pujol (1998; ref. Moreiro, 2000) categoriza los SVA en función de cuatro perspectivas diferentes: a) servicios de comunicación y acceso selectivo a la información a través del correo electrónico; b) acceso a información retrospectiva (hemerotecas); c) consulta de recursos informativos elaborados por el 
propio medio en forma de documentos secundarios - dossieres, bases de datos bibliográficas, directorios de entidades o diccionarios biográficos -; y d) acceso a recursos externos por medio de la elaboración de directorios selectivos.

Es evidente que el vertiginoso desarrollo de este entorno hace que los escenarios evolucionen muy rápidamente. Así pues, en segmentos de tiempo muy cortos podemos ver transformado un portal completamente en otro diferente; no solo con un nuevo diseño, sino con nuevas características en sus productos y servicios. Esta variación constante implica que cualquier identificación y posterior clasificación de los mismos es susceptible de modificación. En la tabla III se pretende destacar el mayor número de estos servicios en función de ocho categorías: acceso a la información, interactivos, participación, multimedia, personalizados, entretenimiento, comerciales y complementarios.

\begin{tabular}{|l|l|}
\hline SVA & \multicolumn{2}{l|}{ Tipología } \\
\hline $\begin{array}{l}\text { Acceso } \\
\text { a la información }\end{array}$ & $\begin{array}{l}\text { Ayuda. Barra de navegación/Página de inicio. Buscador } \\
\text { noticias. Buscador web. Hemeroteca. Herramientas. Mapa } \\
\text { web. Portales verticales. Canales. Últimas noticias. }\end{array}$ \\
\hline Interactivos & Chat. Foros. Webmail. \\
\hline Participación & $\begin{array}{l}\text { Cartas al director. Concursos. Encuestas. Envío de relatos. } \\
\text { MailNew a un amigo. Página abierta. Postales. Sugerencias. }\end{array}$ \\
\hline Multimedia & $\begin{array}{l}\text { Fotografías. Gráficos interactivos. Radio digital. Vídeos. } \\
\text { Televisión digital. }\end{array}$ \\
\hline Personalizados & $\begin{array}{l}\text { Alertas. MailNews. Mi diario. Mi portada. Recordatorios } \\
\text { (eventos). WAPnews. Mascotas virtuales. }\end{array}$ \\
\hline Entretenimiento & Horóscopos. Juegos/Pasatiempos. Webcam. \\
\hline Comerciales & $\begin{array}{l}\text { Tienda/s. Viajes online. Clasificados. Venta de contenidos } \\
\text { (e-contents). Banca online. }\end{array}$ \\
\hline Complementarios & Diccionario. Enlace a otros medios. PDAnews. Traductor. \\
\hline
\end{tabular}

Tabla III. SVA en los portales periodísticos

1. SVA de acceso a la información: En los portales periodísticos, al igual que en cualquier portal o sitio web, tan importante es la información contenida en ellos, como el acceso que se tenga. ¿Qué utilidad tendría ofrecer una información depurada y actualizada, si el usuario-cliente o ciber-lector no sabe llegar hasta ella? Por ello, estos SVA se consideran esenciales para el acceso a las distintas 
informaciones y productos que ofrecen este tipo de portales. Entre ellos cabe destacar la ayuda, el buscador de noticias, la hemeroteca, etcétera.

2. SVA Interactivos: Un SVA interactivo es aquel que permite una interrelación dinámica y frecuente entre el medio y el lector. Estos servicios están orientados a cubrir las necesidades de comunicación de los lectores. La oferta que los portales periodísticos brindan de este tipo de servicios se corresponde en su mayoría a SVA ya clásicos en los portales generales de Internet, tales como chat, foros y webmail.

3. SVA de participación: Éstos se refieren a aquellos servicios que posibilitan la participación del lector. En este caso también se produce una interacción como en el caso de los SVA anteriores, pero aquí es de manera estática, ya que están sometidos a la estructura predeterminada del servicio, y su uso es menos frecuente. Estos SVA están orientados a generar en el lector un sentimiento de protagonismo propiciado por la invitación a participar en el diario. Pero, a diferencia de los interactivos, están en la mayoría de los casos estrechamente vinculados con la información estrictamente periodística tratada en el portal. En este grupo se incluirían secciones como las cartas al director, mailnew a un amigo, encuestas, etc.

4. SVA multimedia: Este grupo de servicios están orientados, principalmente, a reforzar la información textual de las noticias aprovechando la interacción de medios y formatos que brinda Internet. Estos servicios aportan valor añadido a la noticia textual en la medida que ésta puede ser ampliada y documentada, de tal forma que ayude al lector a alcanzar una interpretación más precisa. Es el caso de las fotografías, gráficos interactivos, etcétera.

5. SVA personalizados: Este tipo de servicios están caracterizados por establecer una serie de perfiles de usuario o lector, que facilitan una difusión selectiva de información periodística. Estos SVA solicitan al lector, a priori, una serie de datos sobre edad, sexo, domicilio, preferencias informativas, formatos de presentación, etc., con el fin conformar un perfil personalizado. A este grupo pertenecen las alertas, WAPnews, mascotas virtuales, etcétera.

6. SVA de entretenimiento: En este grupo se incluyen aquellos servicios destinados al ocio y a la diversión del usuario, constituyendo atractivos reclamos del portal periodístico. Por ello, algunos de ellos varían de forma y contenido de forma muy continua. Destacan entre ellos los pasatiempos, juegos, horóscopos o el webcam.

7. SVA comerciales: En esta categoría se agrupan servicios pertenecientes o relacionados con el comercio, es decir, con la compra, venta o permuta de géneros o mercancías. Se trata de espacios que los portales destinan a transacciones entre las empresas y los internautas, como son las tiendas, viajes, banca, etc., y, entre los mismos internautas, como son los casos de las subastas, clasificados, rastros, etc. 
8. SVA complementarios: En esta categoría se concentran aquellos servicios que no han sido agrupados en las categorías anteriores, pero que constituyen un apoyo a la información periodística y al resto de SVA presentes en los portales periodísticos, complementándolos en una serie de aspectos puntuales. Fundamentalmente consisten en fuentes de información de tipo referencial, destacando los diccionarios, traductores, PDAnews, etcétera.

\section{Grado de implantación de los SVA}

Con la finalidad de identificar el grado de desarrollo de los portales periodísticos en España, se llevó a cabo una investigación en la cual se eligió una muestra de diecisiete portales pertenecientes a importantes cabeceras de nuestro país, de las que se analizaron, entre otros aspectos, los SVA más frecuentes en los principales portales periodísticos españoles. Los periódicos analizados fueron ABC, Hoy, La Verdad, La Vanguardia, Mundo Deportivo, Diario Información, Faro de Vigo, La Nueva España, Levante Digital, 5Días, As, El País, El Mundo, Expansión Directo, Marca, El Periódico y Sport.

La tabla IV recoge el grado de desarrollo de los distintos tipos de SVA presentes en los portales periodísticos.

\begin{tabular}{|l|c|c|c|c|}
\hline \multicolumn{1}{|c}{ SVA } & Media & Máx. & \multicolumn{1}{c|}{ Desarrollo } & Portal \\
\hline Acceso & 5,11 & 9 & $56 \%$ & El Mundo, El País, La Verdad \\
\hline Interactivos & 1,70 & 3 & $56 \%$ & ABC, Marca \\
\hline Participación & 3,94 & 8 & $49 \%$ & La Vanguardia \\
\hline Multimedia & 2,23 & 5 & $44 \%$ & El País \\
\hline Personalizados & 1,52 & 7 & $21 \%$ & EI País \\
\hline Entretenimiento & 1,17 & 3 & $39 \%$ & La Verdad \\
\hline Comerciales & 1,82 & 5 & $36 \%$ & 5Días, As, El Mundo, El País \\
\hline Complementarios & 1,29 & 4 & $32 \%$ & \\
\hline
\end{tabular}

Tabla IV. Desarrollo de los distintos tipos de SVA en los portales periodísticos españoles

La media equivale al cociente establecido entre el total de SVA presentes en una categoría determinada y el número de portales analizado (17). La segunda columna recoge el número de SVA identificados en cada categoría, el porcentaje de la tercera columna refleja el grado de desarrollo de esta categoría - expresada 


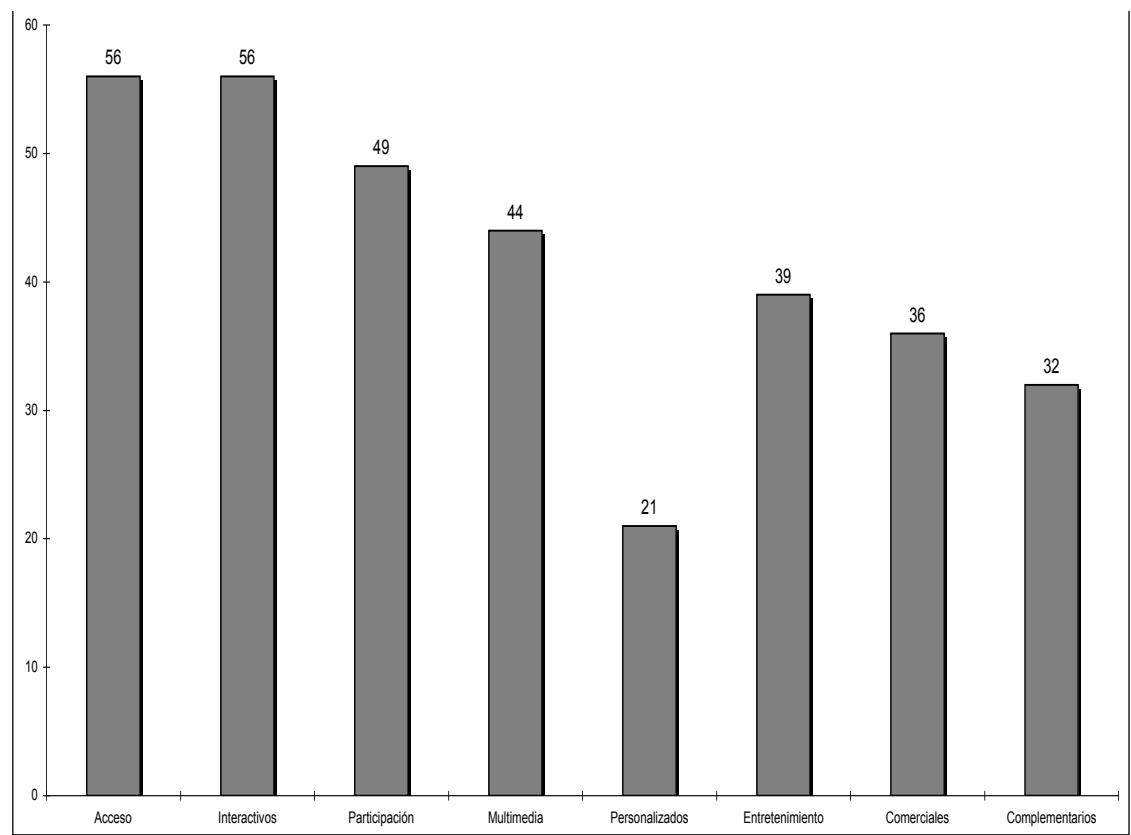

Figura 2. Desarrollo de los SVA en los portales periodísticos españoles

como la proporción de SVA desarrollados sobre el máximo posible - y la última columna indica los portales periodísticos que presentan el mayor número de servicios en cada categoría. Los datos recogidos en esta tabla permiten la construcción de la gráfica de la figura 2. En esta gráfica podemos observar como uno de los resultados más destacados del estudio el predominio de los SVA de acceso a la información y de los interactivos, seguidos muy de cerca por los de participación. Para el resto, los valores medios de implantación y desarrollo no llegan a alcanzar el cincuenta por ciento.

\section{Conclusiones}

El portal periodístico consituye el máximo punto evolutivo del periodismo electrónico actual, dentro del proceso de sinergia entre los medios de comunicación e Internet. El portal periodístico está vinculado a los medios de comunicación social tradicionales o a los desarrollados específicamente para la web. Su eje principal es el contenido, el cual es canalizado a través de los productos informativos, productos documentales y servicios de valor añadido.

Frente a los productos informativos y los productos documentales, los servicios de valor añadido son los elementos más consolidados y frecuentes en los 
portales periodísticos, debido fundamentalmente a su amplia gama y popularidad entre los usuarios. Se han identificando ocho categorías de SVA: acceso a la información, interactivos, participación, multimedia, personalizados, entretenimiento, comerciales y complementarios. Los más consolidados son los pertenecientes a las categorías de acceso a la información e interactivos, seguidos muy de cerca por los de participación. Los portales periodísticos que destacan en la categoría de SVA de acceso a la información son los pertenecientes a los diarios El Mundo, El País y La Verdad. En el caso de los SVA interactivos sobresale ABC y Marca. Con respecto a los SVA de participación destaca La Vanguardia.

\section{Referencias}

Alguacil, D. (2000). Contenidos: ¿la clave para vender? // Todo web business: La revista de los negocios on-line. 10 (agosto 2000). URL: $<\mathrm{http} / / / \mathrm{www}$.webbusinessonline.com/10/afondo.html>. Consultado: 2002-09-10.

Armentia, J. I.; Elexgaray, J.; Pérez, J. C. (1999). Diseño y periodismo electrónico. Bilbao: Servicio Editorial, Universidad del País vasco, 1999.

Baró, J.; Ontalba, J. A. (2001). Portales españoles: ¿demasiados productos para pocos clientes?. // La Universidad Virtual. Barcelona: Universidad Oberta, agosto 2001. URL: $<$ http://www.uoc.edu/web/esp/art/uoc/0107029/portales.html>. Consultado: 2002-10-08.

Braa, K.: et al. (2000). Planet Internet. // Challenges Facing Informations. Annual International Conference on Information Systems (Helsinki, Finland, December 13-16, 1998). Keuruu, Finland, 2000. URL: $<$ http://iris22.it.jyu.fi/iris22/pub/Braa_IRIS22.pdf>. Consultado: 2002-07-02.

Canga Larequi, J. (1999a). Diarios digitales: apuntes sobre un Nuevo Medio. Bilbao: Servicio Editorial. Universidad del País vasco, 1999.

Canga Larequi, J.; et al. (1999b). ¿Quién teme al periódico digital?. // Revista latina de comunicación social. 17 (marzo 1999). URL: $<$ לttp://www.ull.es/publicaciones/latina/a1999hmy/89pvasco.htm>. Consultado: 2002-08-07.

Canga Larequi, J. (2000). Periodismo e Internet: nuevo medio, vieja profesión. // Estudios sobre el mensaje periodístico. 7 (2001). URL: <http://www.ucm.es/info/periol/Period_I/EMP/Numer_07/7-3-Pone/7-3-02.htm>. Consultado: 2002-08-07.

Cely Álvarez, A. M. (1999). Elementos para caracterizar los nuevos medios de comunicación. // Revista latina de comunicación social,.19 (julio 1999). URL: <http://www.ull.es/publicaciones/ latina/biblio/cartagena/68acely.htm>. Consultado: 2002-04-27.

Delgado Soler, C. (1999). Documentación de prensa. // En: García Gutiérrez, A. (ed). Introducción a la Documentación Informativa y Periodística. MAD: Sevilla, 1999. 450-472.

Díaz Noci, J. (1997). Tendencias del periodismo electrónico: Una aproximación a la investigación sobre medios de comunicación en Internet. // Revista de estudios de comunicación. 2 (mayo 1997). URL: <http://www.ehu.es/zer/zer2/6artdiaz.html>. Consultado: 2002-02-10. 
Díaz Noci, J.; Meso Ayerdi, K. (1999a). Periodismo en Internet: modelos de prensa digital. Bilbao: Servicio Editorial, Universidad del País Vasco, 1999.

Díaz Nosty, B. (1999b). Innovación y tendencias en el ámbito de los contenidos periodísticos en Internet. // Revista de periodismo digital. 1 (jun. 2002). URL: $<$ http://teleline.terra.es/personal/simonp/coranto/jun2000/art5.htm>. Consultado: 200204-30.

Fuentes i Pujol, M. E. ; et al. (1998). La prensa española en Internet: análisis de los servicios de valor añadido. // Los sistemas de información al servicio de la sociedad. Valencia: FESABID, 1998. 281-292.

López Carreño, Rosana ; Martínez Méndez, Fco. Javier. (2002). La importancia de los productos documentales dentro de la gestión de los e-contents en los portales periodísticos. [En CD-ROM]. Valencia: CALSI Workshop, 2002.

Moreiro González, José Antonio (coord.) (2000). Manual de documentación informativa. Madrid: Ediciones Cátedra, 2000.

Pablos Coello, J. M. (2001). Necesidad de aclarar conceptos y terminología sobre Internet. // Estudios sobre el mensaje periodístico. 7 (2001). URL: <http://www.ucm.es/info/perioI/Period_I/EMP/Numer_07/7-3-Pone/7-3-04.htm>. Consultado: 2002-08-07.

Palazón Meseguer, A. (2001). Comunicación web: el valor de los contenidos de la Red. // Comunicar: Revista Científica de Comunicación y Educación. 17 (2001) 93-96.

Valdés Abreu, M. C. (1999). Consideraciones generales en torno al valor añadido de la información. // ACIMED. 7:1 (1999) 8-14. URL: <http://bvs.sld.cu/revistas/aci/vol7_1_99/aci02199.htm>. Consultado: 2002-06-27.

Zárraga, J.L. (1997). Los medios de comunicación en Internet. // Madrid: ARGO Redes y Servicios Telemáticos S.A., 1997. URL: $<$ http://www.argo.es/medios/ponencia.html>. Consultado: 2002-06-04. 